\title{
SERUM LIPIDS: INTERACTIONS BETWEEN AGE AND MODERATE INTENSITY EXERCISE
}

\author{
Kay VAN DER EEMS* and A. H. ISMAIL†
}

Department of Physical Education, Health and Recreation, Purdue University, West Lafayette, IN 47907

\section{ABSTRACT}

The purpose of this study was to examine relationships between age and selected serum lipids and lipoproteins in women before and after a physical fitness programme. Twenty females 27.59 years of age who had participated in the Purdue University Physical Fitness Programme were selected and placed into one of two groups: "junior" (mean age 34, all under 40 yrs) or "senior" (mean age 50 , all over 43 ).

A two way factorial design was used to study differences in serum triglycerides (TG), total cholesterol (TC), low density lipoprotein cholesterol (LDLC), high density lipoprotein cholesterol (HDLC), and the risk ratios TC/HDLC and LDLC/HDLC associated with physical fitness and the eight month physical fitness programme. The ability of the biochemical variables to discriminate between the age groups was investigated using discriminant function analyses.

The analyses of variance indicated that although the two age groups were matched on the basis of a multivariate physical fitness score (Ismail et al, 1965) the older group was heavier $(p<0.05)$, and had higher systolic and pulse pressures ( $p<0.05)$. Both groups increased their physical fitness score from pre to post programme $(p<0.01)$.

No significant age related biochemical differences were noted in the univariate analyses; however, in the discriminant function analyses the biochemical variables significantly discriminated between the two groups before, but not after the programme. A decrease in serum triglycerides was observed in the more highly fit women in each age group.

These findings suggest that moderate levels of physical activity may help to counteract some of the undesirable changes in the lipid profile associated with age.

Key words: Lipids, Women, Age, Exercise.

\section{INTRODUCTION}

Ageing in women is associated with little change in the serum concentration of high density lipoprotein (HDL) (Heiss, 1980). At the same time total cholesterol and triglycerides increase (Hallberg, 1967), with concomitant increases in the cholesterol content of the more atherogenic low density and very low density lipoproteins (Dedonder-Decoopman, 1980). These findings suggest that part of the increased cardiovascular risk associated with ageing in women is due to a more atherogenic serum lipid profile.

Physical activity is thought to have an antiatherogenic influence on the serum lipids and lipoproteins (Wood, 1979; Cauley, 1982; Zung Vu Tran, 1983). Though the influence of physical activity in women is probably smaller than that observed in men (Heiss, 1980; Moll, 1979), the influence of exercise on the relationship between serum lipids and age deserves consideration. Highly active middle-aged women may experience a lesser increase in triglycerides with age and carry a greater portion of their serum cholesterol on HDL than sedentary women (Wood, 1977). The purpose of this study was to investigate relationships between age and the serum lipid profile in women before and after an eight month physical fitness programme of moderate intensity.

\section{PROCEDURE}

The subjects for this investigation were healthy females ages 28-59 selected from the participants in the Purdue University Physical Fitness Program. The physiological data considered included age, height weight, body composition as estimated using skinfold measurements (Wilmore, 1970), heart rate and blood pressure following a ten minute rest, and maximal oxygen consumption capacity as estimated from a

\section{-Present address:}

Dept. of Human Nutrition and Food Mgmt.,

265 Campbell Hall,

1787 Neil Avenue,

The Ohio State University,

Columbus $\mathrm{OH} 43210$

tDeceased, 1984 submaximal treadmill test. The fitness score of Ismail et al (Ismail, 1965) was used to obtain an overall measure of physical fitness.

The biochemical variables considered were total cholesterol (TC) and triglycerides (TG) as determined on the Technicon Autoanalyzer II system (Technicon Instruments Co. Tarrytown, NY), HDLC as determined by the procedure of Abbott Laboratories (Abbott Laboratories Diagnostic Division, S. Pasadena, CA), Iow density lipoprotein cholesterol (LDLC) as estimated by the indirect Friedwald procedure (Friedwald, 1972), and the ratios TC/HDLC and LDLC/HDLC.

The Purdue University Physical Fitness Program met three times per week for 90 minutes. It began in early September and ended in early May. Each session began with an informal warm up period. The entire group then participated in $\mathbf{3 0}$ min of rhythmic calesthenics. This was followed by a short period of relaxation training. The next 15-30 min consisted of walking or jogging at 60-70 percent of the individual's heart rate reserve and a five minute cool down period.

The subjects were placed into two age groups: "senior" and "junior". The "junior" group consisted of ten women aged between 27-39 years (mean age 34) and the "senior" group of ten women aged between $43-49$ years (mean age 50 ). The age groups were equated in physical fitness using a multivariate physical fitness index (Ismail, 1965). These two groups were retained throughout the study. The data were examined using an analysis of variance for a two way nested factorial design. Stepwise discriminant function analyses were utilised to determine the ability of the biochemical variables to discriminate between the age groups at both pre and post program.

\section{RESULTS}

The results for the analyses of variance are found in Table I. Though the two age groups were matched on the basis of the physical fitness score, several physiological differences between the age groups were apparent. Systolic blood pressure was higher in the senior group ( $p<0.05$ ). This difference was accompanied by a significant difference in pulse pressure ( $p<0.05$ ) and in body weight $(p<0.05)$. No significant differences for the biochemical variables were detected.

Three significant changes were observed from pre to post programme. Maximal oxygen consumption did not change; however physical fitness score increased in both groups $(p<0.01)$. A significant decrease in percent fat occurred $(p<0.05$ ). This was due mainly to changes in the younger group. A decrease in serum triglycerides was also noted ( $p<0.01$ ). This resulted from decreases in only the more highly fit women in each group.

The results for the discriminant function analyses are found in Table II. The variables entered into the discriminant function analyses 
Table I Pre and post programme age group means and $F$ values from the analysis of variance.

\begin{tabular}{|c|c|c|c|c|c|c|}
\hline \multirow[b]{2}{*}{ Variable } & \multicolumn{2}{|c|}{ Pre Programme } & \multicolumn{2}{|c|}{ Post Programme } & \multicolumn{2}{|c|}{ F Values } \\
\hline & Junior & Senior & Junior & Senior & J vs S & Pre vs Post \\
\hline Age & 34 & 50 & 34 & 50 & $44.43^{* *}$ & $6.25^{*}$ \\
\hline PFS & 244 & 247 & 279 & 258 & 0.50 & $10.75 * *$ \\
\hline $\mathrm{VO}_{2}\left(\mathrm{ml} . \mathrm{kg}^{-1} \mathrm{~min}^{-1}\right)$ & 38.4 & 46.5 & 42.0 & 46.3 & 4.16 & 0.39 \\
\hline RHR (bpm) & 71 & 71 & 70 & 73 & 0.25 & 0.12 \\
\hline SBP $(\mathrm{mmHg})$ & 114 & 130 & 114 & 127 & $5.10^{*}$ & 0.76 \\
\hline DBP $(\mathrm{mmHg})$ & 74 & 80 & 73 & 79 & 2.35 & 0.17 \\
\hline$P P(\mathrm{mmHg})$ & 40 & 50 & 40 & 48 & $6.15^{*}$ & 0.35 \\
\hline Wt $(\mathbf{k g})$ & 56 & 66 & 57 & 66 & $6.48^{*}$ & 0.35 \\
\hline$\%$ Fat & 27 & 29 & 24 & 29 & 3.20 & $5.96^{*}$ \\
\hline$T C(\mathrm{mg} / \mathrm{d} /)$ & 189 & 221 & 191 & 201 & 2.96 & 2.11 \\
\hline TG (mg/dl) & 83 & 85 & 60 & 71 & 0.47 & 9.43 * * \\
\hline HDLC (mg/di) & 52 & 63 & 56 & 61 & 2.84 & 0.06 \\
\hline LDLC (mg/dl) & 118 & 141 & 124 & 125 & 1.08 & 0.77 \\
\hline TC/HDLC & 3.88 & 3.54 & 3.58 & 3.41 & 0.77 & 1.05 \\
\hline LDLC/HDLC & 2.51 & 2.34 & 2.35 & 2.15 & 0.53 & 0.84 \\
\hline
\end{tabular}

*p $<0.05, * * p<0.01$

were TC, TG, HDLC, TC/HDLC and LDLC/HDLC. At the pre test two variables, TC and TC/HDLC, significantly discriminated between the two groups ( $p<0.05$ ) with seventy percent correct classification. The older group had higher TC and surprisingly, a lower score on the cardiovascular risk indicator TC/HDLC. At the post test none of the biochemical variables significantly differentiated between the junior and senior groups.

Table II Pre programme stepwise discriminant function analyses for the biochemical variables.

\begin{tabular}{llccc}
\hline Step & $\begin{array}{c}\text { Variable } \\
\text { Entered }\end{array}$ & $\begin{array}{c}\text { Wilks } \\
\text { Lambda (a) }\end{array}$ & $\begin{array}{c}\text { Canonical Discrim. } \\
\text { Function Coeff. (b) }\end{array}$ & Sign. \\
\hline 1 & TC & 0.75 & 0.98 & 0.93 \\
2 & TC/HDLC & 0.68 & -0.55 & 0.04 \\
\hline
\end{tabular}

Percent of cases correctly classified: $70, p<0.04$.

(a) indicates the proportion of the total variance unaccounted for (Kerlinger and Pedhazur, 1973).

(b) indicates the maximum possible correlation between the variable entered, and the age classification (ibid.).

\section{DISCUSSION}

Though the age groups were matched in physical fitness score several physiological changes which are reported to occur with age in women were detected. Systolic blood pressure was higher in the older group. Pulse pressure, which was correlated with systolic blood pressure at both testing periods $(r=0.84$, $p<0.001)$, was also higher in the older women.

Though the age groups were not different in height, the older group was significantly heavier than the younger group. Overall, the age groups were not significantly different in body composition. The junior group was slightly below the reported age group average of $28.6 \%$ fat for women aged 30 . 40. The older group was more substantially below the average of $34.4 \%$ fat for women aged $40-50$ (Katch and McCardle, 1977).

The significant decrease in percent fat from pre to post test appears to be due mainly to changes in the younger group. Though the interaction between age and testing period was not significant in the analysis of variance, the non parametric Wilcoxon ranked sign test revealed that the young group changed significantly from pre to post test while the old group did not. This result may be the result of selecting older women who were less fat with respect to their age group average and thus more resistant to further decreases than the young group. Another possibility is that the older group did not do as much absolute work as the younger group.
Both groups significantly increased their physical fitness score from pre to post programme. However, only one of the components of the physical fitness score, body composition, changed significantly. This finding suggests that multidimensional indices of fitness status may be more powerful measures of physical fitness than the conventional single variable indicators.

The biochemical differences between the age groups were small. In the univariate analysis the increases in TG, TC, and LDLC reported to occur with age in women were not detected. In a similar study considering middle aged men Ismail and Montgomery reported similar findings (Ismail and Montgomery, 1979). This finding supports the suggestion that physical activity may counteract age related increases in these variables. However, the small size of this study must be considered when interpreting this result.

The more powerful discriminant function analyses detected significant differences between the age groups before, but not after the fitness programme. At the pre test the older group had higher TC but surprisingly, a lower ratio of TC/HDLC. Higher TC but lower cardiovascular risk in older women compared to younger women of the same fitness level suggests that physical activity may play a role in decreasing cardiovascular disease risk in older women. At the post test the biochemical variables did not discriminate between the two groups. For both TC and TC/HDLC the group with the least desirable score had improved, though not significantly in the univariate analysis.

The only significant biochemical change from pre to post programme was a decrease in triglycerides in only the more highly fit women in each age group. The more highly fit women would have been able to train at a greater absolute intensity for a longer time period. This finding supports the suggestion that a minimal threshold level of physical activity may be necessary to influence the serum lipids (Paffenberger et al, 1978; Cauley, 1982). Further research to clarify the intensity, duration, and frequency of exercise needed to bring about these changes in young and older women is needed.

In conclusion, this investigation provides evidence that even moderate levels of physical activity are associated with beneficial changes in the lipid profiles of middle aged women.

\section{References}

Cauley, J. A Laporte, R. E., Kuller, L. H. and Black-Sandler, R., 1982 "The epidemiology of high density lipoprotein cholesterol levels in post-menopausal women". J.Gerontol. 37: 10. 
Dedonder-Decoopman, E. Fievet-Desreumaux, C., Campos, E., Moulin, S., Dewailly, P., Sezille, G. and Jaillard, J., 1980 "Plasma levels of VLDL + LDL-C, HDL-C, triglycerides, and apoproteins B and A-1 in a healthy population". Artherosclerosis 37: 559 .

Friedwald, W. J. Levy, R. J, and Fredrickson, D. S., 1972 "Estimation of plasma LDL concentration without use of the preparative ultracentrifuge". Clin.Chem. 18: 499.

Hallberg, L. and Svanborg, A., 1967 "Cholesterol, phospholipids, and triglycerides in plasma in $\mathbf{5 0}$ year old women". Acta Med.Scand. 181: 185

Heiss, G., 1980 "The epidemiology of plasma high density lipoprotein cholesterol levels: the lipid research clinics program prevalence study summary". Circulation IV Suppl: 116.

Ismail, A. H. Falls, H. B. and Macleod, D. F., 1965 "Development of a criterion for physical fitness tests from factor analysis results". J.Appl.Physiol. 20: 991.

Ismail, A. H. and Montgomery, D. L., 1979 "The effect of a four month physical fitness program on a young and an old group matched for physical fitness". Eur.J.Appl.Physiol. 40 (137): 144.
Katch, F. I. and McCardle, W. D., 1977. Nutrition, Weight Control, and Exercise. Houghton Mifflin Co., Boston, Mass.

Kerlinger, F. N. and Pedhazur, E. J., 1973. Multiple Regression in Behavioral Research. Holt, Rinehart and Winston, New York.

Moll, M. E. Williams, R. S Lester, R. M. Quarfordt, S. H, and Wallace, A. G., 1979 "Cholesterol metabolism in nonobese women: failure of physical conditioning to alter levels of high density lipoprotein cholesterol". Atherosclerosis 34: 159.

Wilmore, J. H. and Behnke, A. R., 1970 "An anthropometric estimation of body density and lean body weight in young women". Am.J. Clin.Nutr. $23(3): 267$

Wood, P. D., Haskel, W. L., Stern, M. P., Lewis, S. and Perry, C., 1977 "Plasma lipoprotein distributions in male and female distance runners". Ann. N.Y.Acad.Sci. 748.

Tran, Z. V., Weltman, A., Glass, G. V. and Mood, D. P., 1983 "The effects of exercise on blood lipids and lipoproteins: a meta-analysis of studies"'. Med.Sci.Sport Ex. 15 (5): 393.

\title{
APPRECIATION
}

At some time in our lives many of us are fortunate enough to encounter an individual whose outstanding ability and genuine concern inspire and challenge us to excellence in both personal and professional endeavours. Professor A. H. Ismail was one of those special people. Thus, we note his passing on with deep sadness; and also with sincere appreciation for the insight, encouragement and joy that he brought to our lives.

\author{
British Association of Sport and Medicine \\ Refresher Course in Sports Medicine \\ at Bisham Abbey National Sports Centre \\ 7th to 11 th October, 1985
}

This is a residential course with accommodation for forty people in shared and single rooms. A few places will be available for non-residents either for the full five days or on a sessional basis.

Course fee: Residents: $£ 100.00$ includes full board

Non-residents: $f \mathbf{4 0 . 0 0}$ exclusive of any meals.

The course is designed for doctors and physiotherapists who have already been on an Introductory Sports Medicine Course and who are actively involved in the care of sportsmen and women.

Provisional Programme

a.m.

Mon. 7 th Oct. Update on physiology Swimming injuries

Tues. 8th Oct. Therapeutics/doping

Wed. 9th Oct. Psychology in sport

Thurs. 10th Oct. Gymnastic injuries Fri. 11th Oct. Team Dynamics

Applications to: Dr. Peter Thomas, The Health Centre Loddon Hall Road, Twyford Berks. RG10 9JA
Football injuries

Visit to Farnham

Park Rehabilitation

Unit

The athletes heart

Running injuries 